\title{
Superconducting Properties of the $\eta$-Pairing State in the Penson-Kolb-Hubbard Model
}

\author{
W.R. Czart and S. Robaszkiewicz \\ Institute of Physics, Adam Mickiewicz University \\ Umultowska 85, 61-614 Poznań, Poland
}

\begin{abstract}
The Penson-Kolb-Hubbard model, i.e. the Hubbard model with the pair-hopping interaction $J$ is studied. We focus on the properties of the superconducting state with the Cooper-pair center-of-mass momentum $\boldsymbol{q}=\boldsymbol{Q}$ ( $\eta$-phase). The transition into the $\eta$-phase, which is favorized by the repulsive $J(J<0)$ is found to occur only above some critical value $\left|J_{\mathrm{c}}\right|$, dependent on band filling, on-site interaction $U$ and band structure, and the system never exhibits standard BCS-like features. This is in obvious contrast with the properties of the isotropic $s$-wave state, stabilized by the attractive $J$ and attractive $U$, which exhibit at $T=0$ a smooth crossover from the BCS-like limit to that of tightly bound pairs with increasing pairing strength.
\end{abstract}

PACS numbers: 74.20.-z, 71.28.+d, 74.25.Ha

\section{Introduction}

The Penson-Kolb-Hubbard (PKH) model is one of the conceptually simplest effective models for studying superconductivity of the narrow band systems with short-range, almost unretarded pairing [1-5]. Its Hamiltonian has the form

$$
H=-t \sum_{\langle i j\rangle \sigma}\left(c_{i \sigma}^{+} c_{j \sigma}+\text { h.c. }\right)+U \sum_{i} n_{i \uparrow} n_{i \downarrow}-\frac{1}{2} J \sum_{\langle i j\rangle}\left(c_{i \uparrow}^{+} c_{i \downarrow}^{+} c_{j \downarrow} c_{j \uparrow}+\text { h.c. }\right),
$$

where $n_{i \sigma}=c_{i \sigma}^{+} c_{i \sigma}, t$ is the single electron hopping integral, $U$ is the on-site density interaction, $J$ is the pair hopping (intersite charge exchange) interaction. $\langle i j\rangle$ restricts the sum to nearest neighbors (nn) and $n=\frac{1}{N} \sum_{i \sigma}\left\langle n_{i \sigma}\right\rangle$. 
The model includes a nonlocal pairing mechanism (the pair hopping term $J$ ) that is distinct from the on-site interaction in the attractive Hubbard (AH) model and that is the driving force of pair formation and also of their condensation.

The PKH model has been investigated only in a few particular limits till now [1-7]. The main efforts concerned the ground state properties of the model in one dimension $(d=1)$ at half-filling $(n=1)[1,2,4]$. For $d$-dimensional hypercubic lattices the ground state diagrams of the half-filled PKH model have been determined by means of the (broken symmetry) Hartree-Fock approximation (HFA) and by the slave-boson mean field method in Ref. [2]. For $d=1$ the diagrams are shown to consist of at least nine different phases including superconducting states, site and bond-located antiferromagnetic and charge-density-waves states, as well as mixed phases with coexisting site and bond orderings. The stability range of the bond-type orderings shrinks with increasing lattice dimensionality and for $d=\infty$ the phase diagram involves exclusively site-located orderings.

Recently, we have studied superconducting characteristics of the PK model [6] and the PKH model [5] in the case of attractive $J(J>0)$, which stabilizes $s$-wave pairing state (S) analogous to that driven by the on-site attraction in the AH model.

In the following we will conclude the properties of the PKH model in the case of repulsive $J(J<0)$. Such an interaction can stabilize the $\eta$-phase, i.e. the superconducting state with the Cooper-pair center of mass momentum $\boldsymbol{q}=\boldsymbol{Q}(\boldsymbol{Q}=\Pi / a, \Pi / a, \ldots)$, with the order parameter $x_{\eta}=\sum_{i} \exp \left(\mathrm{i} \boldsymbol{Q} \cdot \boldsymbol{R}_{i}\right) \times$ $\left\langle c_{i \uparrow}^{+} c_{i \downarrow}^{+}\right\rangle \neq 0[2-4,7]$.

\section{Results and discussion}

We performed an extended analysis of the thermodynamic and electromagnetic properties of the $\eta$-phase of the model for $d$-dimensional hypercubic lattices and arbitrary electron concentration $(0<n<2)$ [8]. In the analysis we used a linear response theory and the electromagnetic kernel was evaluated within the HFA-RPA scheme. The effects of phase fluctuations on $T_{\mathrm{c}}$ for $d=2$ lattices were estimated within the Kosterlitz-Thouless $(\mathrm{K}-\mathrm{T})$ scenario in the same way as it was done previously for the case of S-phase [5, 6]. Moreover, the properties of the zero-bandwidth limit of the model were determined using the variational approach which treats the on-site interaction term exactly.

Below we only quote the main results of this study.

1. The transition into the $\eta$-phase is found to occur only above some critical value $(|J| / B)_{\mathrm{c}}(J<0$ and $B$ is the band width), and the system never exhibits standard BCS-like features. The critical value $J_{\mathrm{c}}$ depends on the form of the density of states (DOS), the value of $U$ and the band filling $n$ (Figs. 1, 2).

2. At any fixed $n$ and $U$ there exists also a second characteristic value of $|J| / B$, which we call $(|J| / B)_{\mathrm{c} 1}$ and $(|J| / B)_{\mathrm{c} 1} \geq(|J| / B)_{\mathrm{c}}$. For all the DOS 


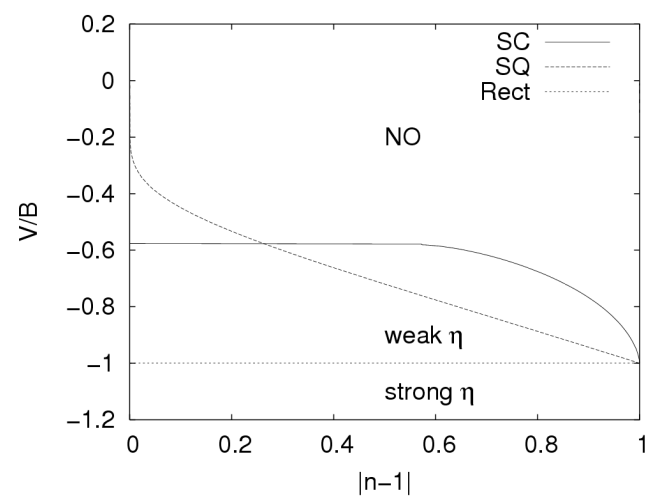

Fig. 1. Ground state phase boundaries between the $\eta$-phase and the NO (normal) state calculated within (broken symmetry) HFA for $d=2$ : SQ (square) $d=3$ : SC (simple cubic) lattices and for rectangular DOS: Rect. $V=J_{0}+U, B=2 z t, J_{0}=z J . z$ is the number of n.n. (strong- $\eta$ and weak- $\eta$ regions are defined in the text).

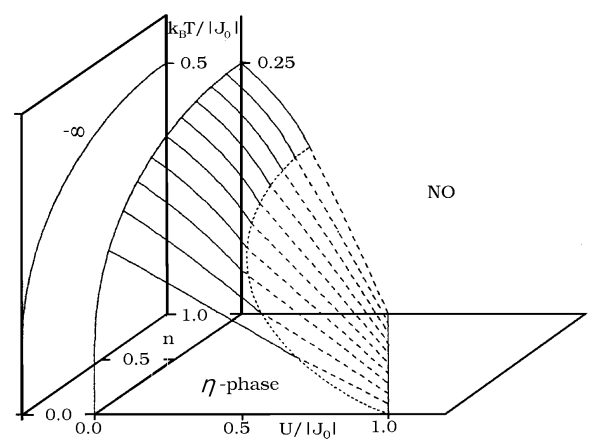

Fig. 2. Finite temperature phase diagram of the PKH model with $J<0$ in the zero-band width limit $(t=0)$ calculated within the variational approach which treats the $U$-term exactly and $J$-term within HFA. The second- and first-order transitions are indicated, respectively, by the solid and the dashed curves. Tricritical points are shown as a dotted line.

considered and $U=0(|J| / B)_{\mathrm{c} 1}=1 / z$ at any $n$. For $|J / B|>(|J| / B)_{\mathrm{c} 1}$ the ground state of the system is characterized by a nonzero gap between the lower and higher quasiparticle band, i.e. $E_{\mathrm{g}}^{\mathrm{m}}(T=0) \equiv \min E_{k}^{+}-\max E_{k}^{-}>0$ and by the order parameter which takes its maximum value (the same as in the zero band width limit) $x_{\eta}^{\max }=\frac{1}{2} \sqrt{n(2-n)}$. We define this state as the strong $\eta$-pairing phase (in analogy with strong ferromagnet). For $|J| / B>(|J| / B)_{\mathrm{c} 1}, T_{\mathrm{c}}>0$ for any $n(0<n<2)$. On the contrary, for $(|J| / B)_{\mathrm{c}}<|J| / B<(|J| / B)_{\mathrm{c} 1}: E_{\mathrm{g}}^{\min } \leq 0$ and $x_{\eta}<x_{\eta}^{\max }$ at $T=0$. Consequently, we define this state as the weak $\eta$-pairing phase (in analogy with weak ferromagnet). This phase exists only in a restricted range of $n$, which shrinks to zero with decreasing $|J|$ (Fig. 1). 
3. For $E_{\mathrm{g}}^{\mathrm{m}}(T)<0$ the quasiparticle DOS in the $\eta$-phase is finite for arbitrary energy but a local minimum in the DOS can occur at the Fermi surface and the system will exhibit a pseudogap behavior [9].

4. The electromagnetic and thermodynamic properties of the $\eta$-phase (for $J<0$ ) become similar to those of the S-phase (for $J>0$ ) only in the large $|J|$ limit: $B /|V| \ll 1,|U| \ll\left|J_{0}\right|$ (compare Figs. 3a, b with Fig. 4 of Ref. [5]). In this limit $H_{\mathrm{c}}^{2}, 1 / \lambda^{2}(0)$, and $T_{\mathrm{c}}$ become proportional to $|J|$, the coherence length $\xi_{\mathrm{GL}}$ tends to a constant value $a / \sqrt{2 z}$, while the Ginzburg ratio $\kappa=\lambda / \xi_{\mathrm{GL}} \sim 1 / \sqrt{|J|}$ and the energy gap $E_{\mathrm{g}}^{\text {min }} \Rightarrow\left|J_{0}\right|$, for any $n$. With decreasing $\left|J_{0}\right| / B$, $\xi_{\mathrm{GL}}$ increases and becomes $n$-dependent, going to infinity at $(|J| / B)_{\mathrm{c}}$ i.e. at the border with NO state.
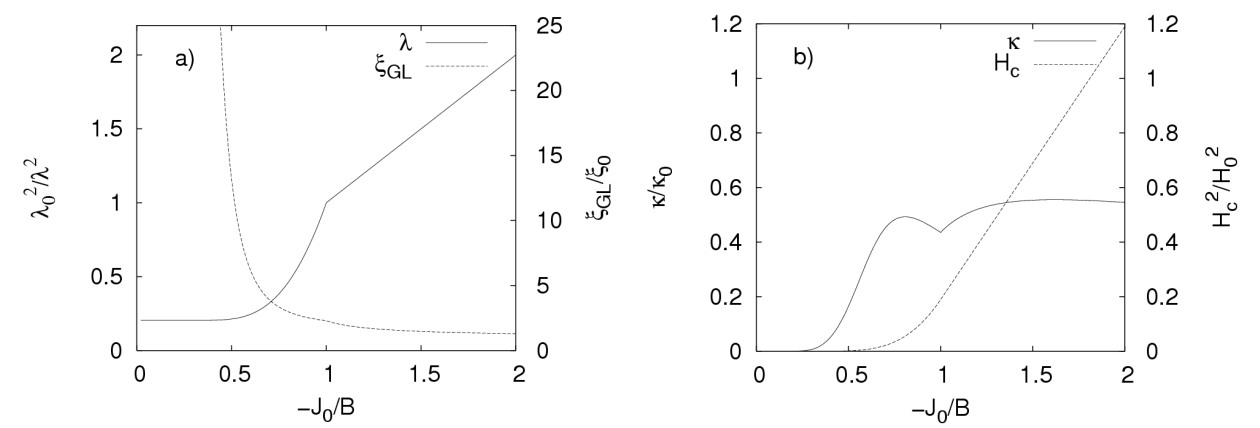

Fig. 3. (a) Inverse square penetration depth $1 / \lambda^{2}\left(\lambda_{0}=\frac{\hbar c}{e} \sqrt{a^{d-2} / 4 \pi B}\right)$ and the Ginzburg-Landau (G-L) coherence length $\xi_{\mathrm{GL}} / \xi_{0}\left(\xi_{0}=a / \sqrt{2 z}\right)$ and (b) the Ginzburg ratio $\kappa / \kappa_{0}\left(\kappa_{0}=\frac{\hbar c}{e} \sqrt{2 / \pi B a^{4-d}}\right)$ and $H_{\mathrm{c}}^{2} / H_{0}^{2}\left(H_{0}^{2}=4 \pi B / a^{d}\right)$ at $T=0$ plotted as a function of $-J_{0} / B$ for SQ lattice and $n=1, U=0$.
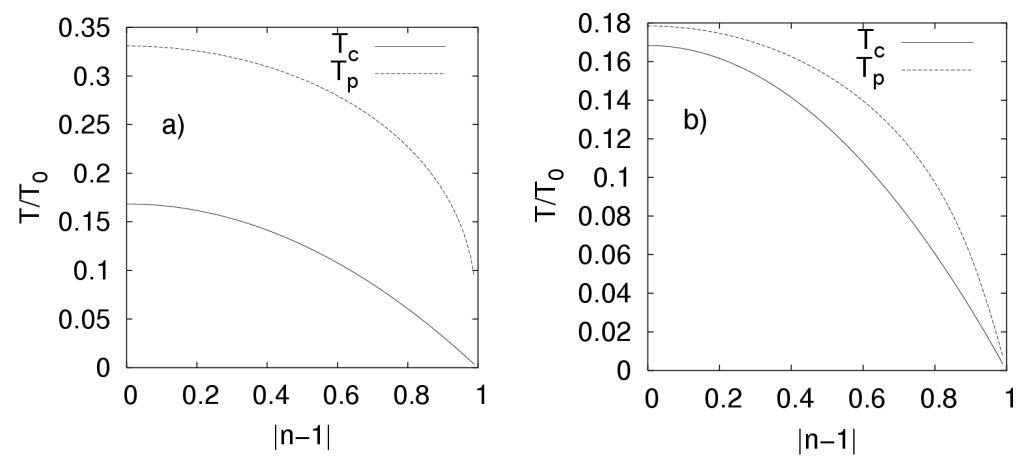

Fig. 4. Transition temperatures for $\eta$-pairing calculated within HFA $\left(T_{\mathrm{p}}\right)$ and within $\mathrm{K}-\mathrm{T}$ approach $\left(T_{\mathrm{c}}\right)$, plotted as a function of $|n-1|$ for SQ lattice: (a) $-J_{0} / B=1.5$ and $U / B=0,(\mathrm{~b})-J_{0} / B=1.5$ and $U / B=0.5\left(T_{0}=B / k_{\mathrm{B}}\right)$. 
5. There are strong effects of phase fluctuations on the $\eta$-phase in $d=2$. As we see from Fig. 4 the $\mathrm{K}-\mathrm{T}$ transition temperature $T_{\mathrm{c}}$ can be substantially lower than $T_{\mathrm{p}}$, which in $d=2$ gives only the estimation of the pair formation temperature.

6. Attractive $U(U<0)$ expands the range of stability of $\eta$-phase at $T=0$ towards lower values of $|J|$. Moreover, the $\eta$-phase can survive also for repulsive values of $U\left(0<U<U_{\mathrm{c}}\right)$. In the narrow band regime $\left(t_{i j} \rightarrow 0\right)$ one finds that the increasing repulsive $U$ changes first the nature of the $\eta$-pairing transition from a continuous to a discontinuous type, resulting in the tricritical point, then it suppresses superconductivity for low $|n-1|$ and finally, for $U /\left|J_{0}\right|>1$ the system remains in a normal state at any $T$ and $n$ (cf. Fig. 2).

\section{Acknowledgments}

We thank R. Micnas and B. Bulka for helpful discussion. This work was supported in part by the State Committee for Scientific Research, grant no. 1 P03B 084 26; 2004-2006.

\section{References}

[1] A. Hui, S. Doniach, Phys. Rev. B 48, 2063 (1993).

[2] S. Robaszkiewicz, B. Bułka, Phys. Rev. B 59, 6430 (1999) and references therein.

[3] F. Dolcini, A. Montorsi, Phys. Rev. B 62, 2315 (2000).

[4] G.I. Japaridze, A.P. Kampf, M. Sekania, P. Kakashvili, Ph. Brune, Phys. Rev. B 65, 014518 (2001).

[5] S. Robaszkiewicz, W.R. Czart, Acta Phys. Pol. B 32, 3267 (2001).

[6] W.R. Czart, S. Robaszkiewicz, Phys. Rev. B 64, 104511 (2001).

[7] S. Robaszkiewicz, W.R. Czart, Phys. Status Solidi B 236, 416 (2003).

[8] W. Czart, S. Robaszkiewicz, in preparation.

[9] M. Mierzejewski, M. Maśka, Phys. Rev. B 69, 054502 (2004). 